\title{
What May Constrain the Success of Indoleamine 2,3-Dioxygenase 1 Inhibitors in Cancer Immunotherapy?
}

\author{
Theodoros Eleftheriadis* \\ Department of Nephrology, Faculty of Medicine, University of Thessaly, Larissa, Greece
}

Keywords: indoleamine 2,3-dioxygenase, cancer immunotherapy, p53, metabolism, T-cell

Indoleamine 2,3-dioxygenase 1 (IDO1) catalyzes the initial rate-limiting step of tryptophan degradation along the kynurenine pathway and suppresses T-cell immune response by two paths; the activation of general control non-derepressible 2 kinase (GCN2K) and aryl-hydrocarbon receptor (AhR). In the microenvironment of the immune response, tryptophan depletion activates GCN2K, which inhibits T-cell proliferation and induces T-cell apoptosis (1). From a teleological point of view, the selection of tryptophan depletion as an immunomodulatory mechanism is ingenious. Tryptophan is an essential amino acid not synthesized by human cells, its concentration in the body is the lowest among all amino acids, and its deprivation due to low intake appears only in 2 days (2). Thus, its depletion in the microenvironment of inflammation can emerge acutely. Interestingly, and indicating the specific role of the above immunomodulatory mechanism, IDO1-induced tryptophan depletion does not affect the other amino acid sensing system, the mammalian target of rapamycin complex 1 (mTORC1), in T-cells (3-5), which is in accordance with studies showing that mTORC1 is sensitive to the depletion of specific amino acids; more precisely of leucine, isoleucine, valine, and possibly arginine, but not of tryptophan (6). In parallel with IDO1-induced GCN2K activation, kynurenine, a derivative of tryptophan degradation, activates $\mathrm{AhR}$, which induces naïve CD4+ T-cell differentiation into regulatory T-cells (7).

The immunosuppressive properties of IDO1 were discovered by the observation that its expression in the placenta contributes to a successful semi-allogenic pregnancy (8). Then it was revealed that inflammatory stimuli induce IDO1 expression in antigen-presenting cells, and the immunosuppressive role of this enzyme has been confirmed in experimental models of autoimmunity and transplantation (9-12).

Indoleamine 2,3-dioxygenase 1 is also expressed in many types of cancer, and the majority of studies suggest that this enzyme plays a significant role in the escape of tumors from immunosurveillance $(13,14)$. More precisely in various types of cancer, IDO1 expression has been confirmed, individually or in combination, in tumor cells, in interstitial cells in lymphocyte-rich areas, and in endothelial cells. In most cases, IDO1 expression seems to be the result of an ongoing immune response by infiltrating T-cells and other immune cells that produce interferon- $\gamma($ IFN- $\gamma)(14,15)$, a cytokine that induces macrophage and dendritic cell (DC) activation and IDO1 expression (13, 14, 16). The infiltrating immune cells fail to eliminate cancer cells because due to accumulated mutations they escape the initial immune response. The persisted immune response results in increased IDO1 expression by tolerogenic DCs, myeloid-derived suppressor cells, and tumor-associated macrophages. Tryptophan depletion and kynurenine production by IDO1 induce more immune cells to become tolerogenic and inhibit effector T-cells, whereas increase regulatory T-cells. Regulatory T-cells by expressing cytotoxic T-lymphocyte-associated-antigen-4 (CTLA-4) inhibit further effector T-cells and increase IDO1 expression in DCs closing a positive feedback loop of immunosuppression (16). However, in a subset of tumors IDO1 is expressed by cancer cells in the absence of any inflammation indicating that it may be the result of oncogenic events and may contribute to escape of tumor by immunosurveillance by preventing T-cell infiltration $(14,15)$. 
Nevertheless, other studies question the role of IDO1 overexpression in the adverse clinical outcome of certain cancers. Ishio et al. found that the recurrence-free survival rate of patients with IDO1-positive hepatocellular carcinoma is significantly higher than that of patients with IDO1-negative hepatocellular carcinoma (17). Takao et al. showed that increased IDO1 protein is related to worse prognosis in patients with serous type, but not with clear cell or endometrioid type of ovarian adenocarcinoma (18). Riesenberg et al. revealed that the expression of IDO1 in tumor endothelial cells correlates with long-term survival of patients with renal cell carcinoma (19). Jacquemier et al. determined that high IDO expression is associated with morphological medullary features and has an independent favorable prognostic value in patients with basal-like breast carcinoma (20). Recently, Heeren et al. showed that in patients with early stage cervical cancer, a marginal IDO expression pattern in the tumor dominantly predicts a favorable outcome, which might be related to IFN- $\gamma$ release in the cervical tumor microenvironment (21).
Most importantly, despite the initial experimental and clinical indications about the efficacy of IDO1 inhibitors in cancer immunotherapy (16), in the recently Incyte's phase III clinical trial, the addition of the IDO1 inhibitor epacadostat in a therapy with the programmed death 1 immune checkpoint inhibitor pembrolizumab, made no difference for the patients with metastatic melanoma receiving both drugs. This failure led three companies to the decision to suspend, cancel, or downsize 13 trials of IDO1 inhibitors in combination with immune checkpoint inhibitors (22).

There are some possible explanations for these disappointing results. First, IDO1 expression, confirmed by either immunohistochemistry or polymerase chain reaction, in a tumor does not necessarily mean that this enzyme is functional. For instance, IFN- $\gamma$ induces both the expression of IDO1 and the production of nitrogen monoxide (NO) in macrophages, but the latter inhibits IDO1 enzymatic activity (23). Also, in an inflammatory environment, both NO and superoxide anion are produced resulting in the generation of peroxynitrite anion, which inhibits by nitration IDO1

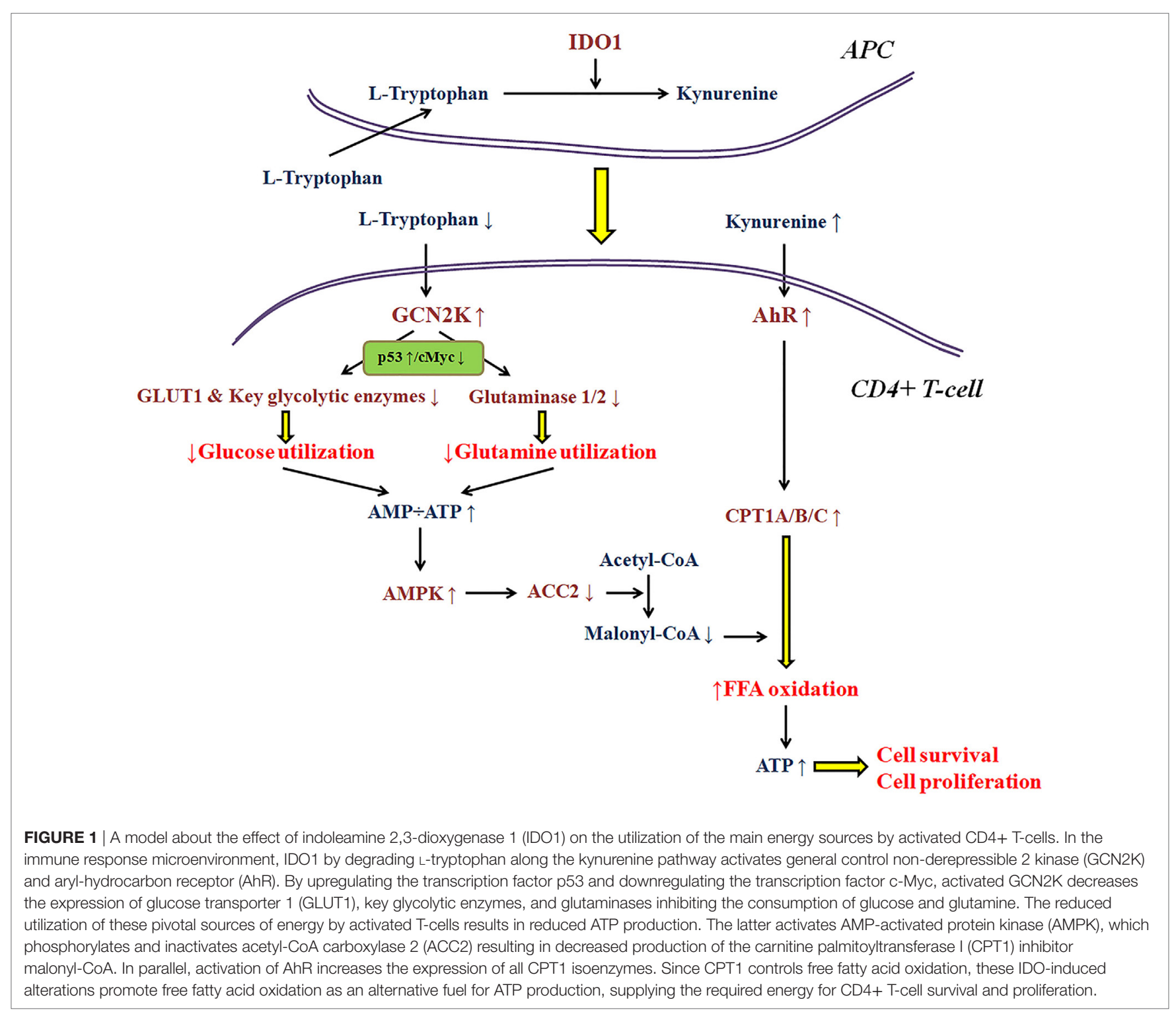


enzymatic activity without affecting its protein level (24). Moreover, phosphorylation of specific IDO1 tyrosine residues blocks its catalytic activity (25). Thus, assessing along with IDO1 expression its enzymatic activity by detecting in the tumors along with IDO1, proteins that are known to be modified or expressed after GCN2K or AhR activation would yield more accurate results about the role of this enzyme in the escape of tumors from immunosurveillance.

In addition, IDO1, by activating GCN2K, alters the metabolism of T-cells, inhibits their proliferation and induces apoptosis in a p53-dependent way $(4,26,27)$. The transcription factor $\mathrm{p} 53$, also known as tumor suppressor p53, inhibits aerobic glycolysis, which characterizes rapidly proliferating cells, and induces cell cycle arrest and/or apoptosis $(28,29)$. Interestingly, activated GCN2K also increases p53 expression in nonimmune cells, such as human aortic endothelial and renal epithelial cells $(30,31)$. The fact that in most the tumors the $\mathrm{p} 53$ pathway is directly or indirectly inactivated (28), offers an advantage in cancer progression. IDO1 expressed by cancer cells or infiltrating immune cells by depleting tryptophan in the local microenvironment activates GCN2K in the T-cells that infiltrate the lesion inhibiting their proliferation and inducing apoptosis. On the contrary, due to the ineffective p53 pathway in the cancer cells, tryptophan depletion does not inhibit tumor growth. Acting in such a way, IDO1 contributes to the escape of cancer from the immunosurveillance. However, in the case of cancer with the intact 153 pathway, the IDO1 expressed by the infiltrating immune cells may be able to activate GCN2K in cancer cells and inhibit tumor progression in a p53-dependent way. In such a case, the administration of an IDO1 inhibitor may decrease the antitumor immune response. Interestingly, in an experimental study, IFN- $\gamma$ exhibited its antiproliferative effects only in cancer cell lines in which it upregulated IDO1 expression with a consequent tryptophan deprivation; suggesting a possible direct antitumor effect of this enzyme in certain types of cancer. However, the p53 pathway was not assessed in the tested cancer cell lines (32). Thus, evaluation of the cancer p53 status before the administration of an IDO1 inhibitor may be vital.

Also, and despite the studies about the role of IDO1 in supporting tumor vessel formation $(33,34)$, the ability of activated GCN2K to induce p53 expression, and possibly cell cycle arrest or apoptosis, in endothelial cells (30), raises questions about the effect of IDO1 inhibition on the required for the tumor progression neoangiogenesis. Interestingly, expression of IDO1 in endothelial cells of renal tumors is associated with a better prognosis (19).

As regards the immunosuppressive properties of IDO1 per se, research in my laboratory, revealed that this enzyme affects T-cell fate at least in part by altering cell metabolism $(3-5,26,35,36)$. Thus, the availability of various nutrients in the microenvironment of the immune response may have a significant impact on IDO1 immunomodulatory properties. Most of the conclusions about the molecular pathways involved in the IDO1-induced immunosuppression were extrapolated under the strictly controlled conditions of cell cultures $(1,7)$. Nevertheless, if a free fatty acid is added in the culture medium, the trend for CD4+ $\mathrm{T}$-cell differentiation toward a regulatory phenotype remains, but the antiproliferative and pro-apoptotic properties of IDO1 disappear $(35,36)$. The reason relies on the effect of IDO1 on T-cell metabolism. As depicted in more detail in Figure 1, depletion of

TABLE 1 | Factors that may limit the anticancer effect of indoleamine 2,3-dioxygenase 1 (IDO1) inhibitors.

The role of IDO1 in the escape of cancer from immunosurveillance may be overestimated

In certain tumors, IDO1 may induce apoptosis of the cancer cells

\section{In certain tumors, \\ ID01 may suppress neoangiogenesis}

The immunosuppressive properties of IDO1 may be overestimated
Most studies assessed only IDO1 expression but not its activity. However, certain conditions that may be present in the cancer microenvironment may inhibit IDO1 activity without affecting its protein level

In human lymphocytes, epithelial and endothelial cells, IDO1 by activating general control nonderepressible 2 kinase (GCN2K) induces p53mediated apoptosis. Thus, in the minority of cancers with an intact p53 pathway, IDO1 expression in the infiltrating immune cells may be beneficial

Although there are studies that support a positive role for IDO1 in tumor neoangiogenesis, the fact that GCN2K activation induces p53-mediated apoptosis in human endothelial cells raises questions. In renal carcinoma, the expression of IDO1 in endothelial cells signifies a worse prognosis

Traditionally, it is thought that IDO1 suppresses T-cells proliferation, induces their apoptosis, and promotes their differentiation toward a regulatory phenotype. However, the presence of free fatty acids in the tumor microenvironment may abolish the antiproliferative and pro-apoptotic properties of IDO1

tryptophan by activating GCN2K inhibits glucose and glutamine catabolism $(3,4,26,36)$. However, kynurenine by activating AhR induces free fatty acid $\beta$-oxidation, which refuels CD4+ T-cells with energy, allowing their proliferation and preventing their apoptosis $(35,36)$. Accordingly, two of the three ways by which IDO1 is supposed to suppress T-cell-mediated immune response may not take place if enough free fatty acids are present in the cancer microenvironment. In such a case, the gain in antitumor immunity by inhibition of IDO1 would be far less than the expected. The data about the concentration of free fatty acids in the various types of cancer are scarce.

In conclusion, there are many aspects to be revealed about the role of IDO1 in the escape of cancer from immunosurveillance (Table 1). Along with tumor IDO1 expression, assessment of its activity may prevent overestimation of its role in the escape of cancer from immunosurveillance. In cancer with an intact p53 pathway, expression of IDO1 by the infiltrating immune cells may exhibit antitumor activity. Also, in an environment relatively rich in free fatty acids the immunosuppressive properties of IDO1 may be decreased considerably, and the gain in antitumor immunity from its inhibition may be less than the expected. The role of IDO1 in tumor neoangiogenesis remains to be better elucidated as well. Administration of IDO1 inhibitors may be beneficial to certain but not all cancers. Beyond tumor IDO1 expression, assessment of other factors such as IDO enzymatic activity, the status of the p53 pathway in the cancer cells, and the availability of free fatty acids in the tumor microenvironment, i.e., the application of a more personalized medicine, may help IDO1 inhibitors to find their place in cancer immunotherapy.

\section{AUTHOR CONTRIBUTIONS}

The author confirms being the sole contributor of this work and approved it for publication. 


\section{REFERENCES}

1. Munn DH, Sharma MD, Baban B, Harding HP, Zhang Y, Ron D, et al. GCN2 kinase in T cells mediates proliferative arrest and anergy induction in response to indoleamine 2,3-dioxygenase. Immunity (2005) 22(5):633-42. doi:10.1016/j.immuni.2005.03.013

2. Ellenbogen MA, Young SN, Dean P, Palmour RM, Benkelfat C. Mood response to acute tryptophan depletion in healthy volunteers: sex differences and temporal stability. Neuropsychopharmacology (1996) 15(5):465-74. doi:10.1016/ S0893-133X(96)00056-5

3. Eleftheriadis T, Pissas G, Yiannaki E, Markala D, Arampatzis S, Antoniadi $\mathrm{G}$, et al. Inhibition of indoleamine 2,3-dioxygenase in mixed lymphocyte reaction affects glucose influx and enzymes involved in aerobic glycolysis and glutaminolysis in alloreactive T-cells. Hum Immunol (2013) 74(12):1501-9. doi:10.1016/j.humimm.2013.08.268

4. Eleftheriadis T, Pissas G, Antoniadi G, Spanoulis A, Liakopoulos V, Stefanidis I. Indoleamine 2,3-dioxygenase increases p53 levels in alloreactive human $\mathrm{T}$ cells, and both indoleamine 2,3-dioxygenase and p53 suppress glucose uptake, glycolysis and proliferation. Int Immunol (2014) 26(12):673-84. doi:10.1093/intimm/dxu077

5. Eleftheriadis T, Pissas G, Antoniadi G, Liakopoulos V, Stefanidis I. Indoleamine 2,3-dioxygenase depletes tryptophan, activates general control non-derepressible 2 kinase and down-regulates key enzymes involved in fatty acid synthesis in primary human CD4+ T cells. Immunology (2015) 146(2):292-300. doi:10.1111/imm.12502

6. Gallinetti J, Harputlugil E, Mitchell JR. Amino acid sensing in dietary-restriction-mediated longevity: roles of signal-transducing kinases GCN2 and TOR. Biochem J (2013) 449(1):1-10. doi:10.1042/BJ20121098

7. Mezrich JD, Fechner JH, Zhang X, Johnson BP, Burlingham WJ, Bradfield CA. An interaction between kynurenine and the aryl hydrocarbon receptor can generate regulatory T cells. J Immunol (2010) 185(6):3190-8. doi:10.4049/ jimmunol.0903670

8. Munn DH, Zhou M, Attwood JT, Bondarev I, Conway SJ, Marshall B, et al. Prevention of allogeneic fetal rejection by tryptophan catabolism. Science (1998) 281(5380):1191-3. doi:10.1126/science.281.5380.1191

9. Gurtner GJ, Newberry RD, Schloemann SR, McDonald KG, Stenson WF. Inhibition of indoleamine 2,3-dioxygenase augments trinitrobenzene sulfonic acid colitis in mice. Gastroenterology (2003) 125(6):1762-73. doi:10.1053/j. gastro.2003.08.031

10. Kwidzinski E, Bunse J, Aktas O, Richter D, Mutlu L, Zipp F, et al. Indolamine 2,3-dioxygenase is expressed in the CNS and down-regulates autoimmune inflammation. FASEB J (2005) 19(10):1347-9. doi:10.1096/fj.04-3228fje

11. Alexander AM, Crawford M, Bertera S, Rudert WA, Takikawa O, Robbins $\mathrm{PD}$, et al. Indoleamine 2,3-dioxygenase expression in transplanted NOD Islets prolongs graft survival after adoptive transfer of diabetogenic splenocytes. Diabetes (2002) 51(2):356-65. doi:10.2337/diabetes.51.2.356

12. Beutelspacher SC, Pillai R, Watson MP, Tan PH, Tsang J, McClure MO, et al. Function of indoleamine 2,3-dioxygenase in corneal allograft rejection and prolongation of allograft survival by over-expression. Eur J Immunol (2006) 36(3):690-700. doi:10.1002/eji.200535238

13. Munn DH, Mellor AL. Indoleamine 2,3-dioxygenase and tumor-induced tolerance. J Clin Invest (2007) 117(5):1147-54. doi:10.1172/JCI31178

14. van Baren N, Van den Eynde BJ. Tryptophan-degrading enzymes in tumoral immune resistance. Front Immunol (2015) 6:34. doi:10.3389/fimmu.2015. 00034

15. Theate I, van Baren N, Pilotte L, Moulin P, Larrieu P, Renauld JC, et al. Extensive profiling of the expression of the indoleamine 2,3-dioxygenase 1 protein in normal and tumoral human tissues. Cancer Immunol Res (2015) 3(2):161-72. doi:10.1158/2326-6066.CIR-14-0137

16. Hornyak L, Dobos N, Koncz G, Karanyi Z, Pall D, Szabo Z, et al. The role of indoleamine-2,3-dioxygenase in cancer development, diagnostics, and therapy. Front Immunol (2018) 9:151. doi:10.3389/fimmu.2018.00151

17. Ishio T, Goto S, Tahara K, Tone S, Kawano K, Kitano S. Immunoactivative role of indoleamine 2,3-dioxygenase in human hepatocellular carcinoma. J Gastroenterol Hepatol (2004) 19(3):319-26. doi:10.1111/j.1440-1746.2003. 03259.x

18. Takao M, Okamoto A, Nikaido T, Urashima M, Takakura S, Saito M, et al. Increased synthesis of indoleamine-2,3-dioxygenase protein is positively associated with impaired survival in patients with serous-type, but not with other types of, ovarian cancer. Oncol Rep (2007) 17(6):1333-9. doi:10.3892/ or.17.6.1333

19. Riesenberg R, Weiler C, Spring O, Eder M, Buchner A, Popp T, et al. Expression of indoleamine 2,3-dioxygenase in tumor endothelial cells correlates with long-term survival of patients with renal cell carcinoma. Clin Cancer Res (2007) 13(23):6993-7002. doi:10.1158/1078-0432.CCR-07-0942

20. Jacquemier J, Bertucci F, Finetti P, Esterni B, Charafe-Jauffret E, Thibult ML, et al. High expression of indoleamine 2,3-dioxygenase in the tumour is associated with medullary features and favourable outcome in basal-like breast carcinoma. Int J Cancer (2012) 130(1):96-104. doi:10.1002/ijc.25979

21. Heeren AM, van Dijk I, Berry DR, Khelil M, Ferns D, Kole J, et al. Indoleamine 2,3-dioxygenase expression pattern in the tumor microenvironment predicts clinical outcome in early stage cervical cancer. Front Immunol (2018) 9:1598. doi:10.3389/fimmu.2018.01598

22. Garber K. A new cancer immunotherapy suffers a setback. Science (2018) 360(6389):588. doi:10.1126/science.360.6389.588

23. Thomas SR, Mohr D, Stocker R. Nitric oxide inhibits indoleamine 2,3-dioxygenase activity in interferon-gamma primed mononuclear phagocytes. J Biol Chem (1994) 269(20):14457-64

24. Fujigaki H, Saito K, Lin F, Fujigaki S, Takahashi K, Martin BM, et al. Nitration and inactivation of IDO by peroxynitrite. J Immunol (2006) 176(1):372-9. doi:10.4049/jimmunol.176.1.372

25. Albini E, Rosini V, Gargaro M, Mondanelli G, Belladonna ML, Pallotta MT, et al. Distinct roles of immunoreceptor tyrosine-based motifs in immunosuppressive indoleamine 2,3-dioxygenase 1. J Cell Mol Med (2017) 21(1):165-76. doi: $10.1111 / \mathrm{jcmm} .12954$

26. Eleftheriadis T, Pissas G, Antoniadi G, Tsogka K, Sounidaki M, Liakopoulos $\mathrm{V}$, et al. Indoleamine 2,3dioxygenase downregulates $\mathrm{T}$ cell receptor complex zetachain and $\mathrm{cMyc}$, and reduces proliferation, lactate dehydrogenase levels and mitochondrial glutaminase in human Tcells. Mol Med Rep (2016) 13(1):925-32. doi:10.3892/mmr.2015.4595

27. Eleftheriadis T, Pissas G, Antoniadi G, Liakopoulos V, Tsogka K, Sounidaki M, et al. Differential effects of the two amino acid sensing systems, the GCN2 kinase and the mTOR complex 1, on primary human alloreactive CD4(+) T-cells. Int J Mol Med (2016) 37(5):1412-20. doi:10.3892/ijmm.2016.2547

28. Brady CA, Attardi LD. p53 at a glance. J Cell Sci (2010) 123(Pt 15):2527-32. doi:10.1242/jcs.064501

29. Labuschagne CF, Zani F, Vousden KH. Control of metabolism by p53 cancer and beyond. Biochim Biophys Acta, Rev Cancer (2018). doi:10.1016/j. bbcan.2018.06.001

30. Eleftheriadis T, Pissas G, Sounidaki M, Antoniadi G, Rountas C, Liakopoulos V, et al. Tryptophan depletion under conditions that imitate insulin resistance enhances fatty acid oxidation and induces endothelial dysfunction through reactive oxygen species-dependent and independent pathways. Mol Cell Biochem (2017) 428(1-2):41-56. doi:10.1007/s11010016-2915-7

31. Eleftheriadis T, Pissas G, Sounidaki M, Antoniadis N, Antoniadi G, Liakopoulos V, et al. Preconditioning of primary human renal proximal tubular epithelial cells without tryptophan increases survival under hypoxia by inducing autophagy. Int Urol Nephrol (2017) 49(7):1297-307. doi:10.1007/ s11255-017-1596-9

32. Ozaki Y, Edelstein MP, Duch DS. Induction of indoleamine 2,3-dioxygenase: a mechanism of the antitumor activity of interferon gamma. Proc Natl Acad Sci U S A (1988) 85(4):1242-6. doi:10.1073/pnas.85.4.1242

33. Pan J, Yuan K, Peng S, Huang Y, Zhang Y, Hu Y, et al. Gene silencing of indoleamine 2,3-dioxygenase hinders tumor growth through angiogenesis inhibition. Int J Oncol (2017) 50(6):2136-44. doi:10.3892/ijo.2017.3975

34. Wei L, Zhu S, Li M, Li F, Wei F, Liu J, et al. High indoleamine 2,3-dioxygenase is correlated with microvessel density and worse prognosis in breast cancer. Front Immunol (2018) 9:724. doi:10.3389/fimmu.2018.00724

35. Eleftheriadis T, Pissas G, Sounidaki M, Tsogka K, Antoniadis N, Antoniadi G, et al. Indoleamine 2,3-dioxygenase, by degrading L-tryptophan, enhances carnitine palmitoyltransferase I activity and fatty acid oxidation, and exerts fatty acid-dependent effects in human alloreactive CD4+ T-cells. Int J Mol Med (2016) 38(5):1605-13. doi:10.3892/ijmm.2016.2750

36. Eleftheriadis T, Pissas G, Liakopoulos V, Stefanidis I. IDO decreases glycolysis and glutaminolysis by activating $\mathrm{GCN} 2 \mathrm{~K}$, while it increases fatty acid oxidation 
by activating AhR, thus preserving $\mathrm{CD} 4+$ Tcell survival and proliferation. Int J Mol Med (2018) 42(1):557-68. doi:10.3892/ijmm.2018.3624

Conflict of Interest Statement: The author declares that the research was conducted in the absence of any commercial or financial relationships that could be construed as a potential conflict of interest.
Copyright () 2018 Eleftheriadis. This is an open-access article distributed under the terms of the Creative Commons Attribution License (CC BY). The use, distribution or reproduction in other forums is permitted, provided the original author(s) and the copyright owner(s) are credited and that the original publication in this journal is cited, in accordance with accepted academic practice. No use, distribution or reproduction is permitted which does not comply with these terms. 